\title{
MAIZ Y POBREZA: ESTUDIO EN OAXACA Y CHIAPAS
}

\begin{abstract}
Javier Becerril García
Doctor en Economía Agrícola por la Universidad de Kiel, Alemania. Maestro en Economía Ecológica por la Universidad Autónoma de Barcelona. Maestro en Urbanismo, Economía y Ambiente por la Universidad Nacional Autónoma de México. Licenciado en Economía por la Universidad Autónoma Metropolitana, unidad Azcapotzalco. El Dr. Becerril García ha sido consultor para organismos internacionales como el Centro Internacional de Mejoramiento de Maíz y Trigo (CIMMyT, Int.) y para la División de Estudios Económicos de Organización sobre Agricultura y Alimentación (FAO) de las Naciones Unidas. Actualmente es profesor-investigador de la Facultad de Economía de la Universidad Autónoma de Yucatán.

javier.becerril@uady.mx
\end{abstract}




\section{1.- CONTEXTO}

El maíz es uno de los tres cereales de mayor importancia en el mundo, seguido del arroz y el trigo (Morris, 1998). La producción de este cereal en México es de suma importancia, tanto económica, social y política (Aquino, 1998; SEP, 2002), dado que el maíz es la base y sustento para millones de habitantes del medio rural y urbano. Su consumo es básicamente en forma de tortillas en toda la geografía nacional. El cereal contribuye en dos tercios de las calorías y un tercio de las proteínas ingeridas en la dieta mexicana (Aquino, 1998). De acuerdo con Morris (1998) el consumo per-cápita es de 127 kilogramos al año aproximadamente. Además, el maíz es un insumo importante para el sector pecuario y la industria del almidón, entre otras industrias.

Por otro lado, en el año 2000, el 19\% de los productores maiceros de subsistencia viven y se desarrollan inmersos en pobreza extrema, es decir, con menos de un dólar al día (CTMP, 2002). Paradójicamente, los productores de subsistencia conservan una riqueza invaluable de biodiversidad, entre ellos destacan los diferentes tipos de maíz, o razas, que se cultivan en ecosistemas heterogéneos, que van de ambientes muy próximos al nivel del mar hasta altitudes superiores a los tres mil metros sobre el nivel del mar, comprendiendo un mosaico muy amplio de microclimas, que van desde muy cálido a muy frío, y de muy húmedo a muy seco (Perales et al., 2003a; Perales et al., 2003b). Por el número de razas, condiciones de producción y otros fundamentos más México es centro de origen y domesticación de este cereal (Brush, 1986; Bellon y Brush, 1994; SEP, 2002).

Actualmente, y derivado de los programas gubernamentales de transferencia de variedades mejoradas de maíz (de polinización abierta e híbridos), los agricultores cuentan con un mayor número de tipos de maíz en sus parecelas (Mauricio Bellon y Alfonso Aguirre, comunicación personal), sin pasar por alto la importancia que ha tomado la industria privada en venta y promoción de variedades mejoradas; así entonces, el número de variedades va desde materiales nativos (criollos), acriollados (generaciones avanzadas de maíces mejorados), variedades de polinización abierta y maíces híbridos (para una mayor descripción de estos materiales ver (Bellon et al., 2003), claro está, desde los primeros inventarios de maíces nativos se han registrado perdidas irreversibles de estos recursos genéticos de variedades nativas, pero también 
se ha registrado un aumento (Ortiga-Paczka, comunicación personal). Es decir, la producción de maíz en manos de pequeños productores de subsistencia es muy compleja y dinámica.

En cuanto a productividad se refiere, a pesar de los diferentes programas de gobierno que se remontan a la década de los 6o's sobre transferencia de tecnología, principalmente paquetes de innovación de tipo: biológico (germoplasma o variedades de maíz mejoradas), químico (fertilizantes, pesticidas y fungicidas), físico (maquinaria que comprende tractores, arado de disco e infraestructura de riego), y de conocimiento (técnicas de labranza, por ejemplo surcado, siembra en camas, etc.), el área plantada con variedades mejoradas es baja y poco tecnificada, pues en 1996 solo 25\% del área total nacional cultivada con maíz (que comprende temporal y riego) se sembró con variedades mejoradas de maíz, es decir, maíces de polinización abierta y maíces híbridos (Morris y López-Pereira, 1999). Adicionalmente, en el ámbito mundial, la productividad maicera en México continua siendo baja, pues alcanza las 2.6 toneladas (t) por hectárea (ha), en Argentina 4.4 t/ha; Chile 8.5 t/ ha y en China $5 \mathrm{t} /$ ha; Mientras que en EE.UU y países de Europa occidental se pueden alcanzar las $10 \mathrm{t} / \mathrm{ha}$ (Morris, 1998a; SIAP, 2007) . Otra característica del agro mexicano es su alta heterogeneidad, es decir, hay productores agrícolas que destinan toda su producción al mercado, tienen acceso a información, tecnología y participan en mercados competitivos, mientras que otro grupo es netamente de subsistencia y su producción es básicamente para autoconsumo, con nulo acceso al mercado y tecnología. Obviamente, esto se debe en mucho a la orografía del país, a la dotación de tierra de los agricultores, la calidad de suelo y acceso a agua para riego, siendo mayoritariamente áreas que dependen de la agua de lluvia y por consiguiente, se aprovecha un solo ciclo agrícola, entre otros muchos más factores.

Adicionalmente, en las últimas décadas México ha llevado a cabo un redimensionamiento del estado en la participación de la economía y reformas estructurales encaminadas a un modelo de corte neoliberal, que se origina en los años ochentas con la incorporación de la economía mexicana al acuerdo general sobre aranceles y comercio (GATT, por sus siglas en Inglés), acentuando el proceso de liberalización de la economía con la firma del tratado de libre comercio con América del norte (TLCAN) en 1994; más la reforma al artículo 27 constitucional (ley agraria y culminación del reparto agrario) en 
1992; la eliminación de los precios de garantía en 1991 para los principales cultivos, entre ellos el maíz. Dentro de las políticas de adelgazamiento del estado destaca la desaparición en 1999 de la compañía nacional de subsistencia populares (CONASUPO) que fungía como centro de acopio y abasto de productos básicos, asumiendo el papel de regulador de los precios de los productos de consumo de la canasta básica de alimentos (Dyer y Yunez-Naude, 2003; Yunez-Naude, 2003), y la desaparición de la productora nacional de semillas (PRONASE) a finales de los años noventa (Aquino, 1998), entre otras reformas estructurales.

En la literatura hay poca evidencia empírica de la relación que existe entre pobreza y el consumo de maíz (en todas sus modalidades: grano, harina, masa, etc.) por parte de los pequeños productores de subsistencia, así entonces, este estudio pretende demostrar esta relación con datos reales recopilados en una encuesta aplicada a 163 productores de maíz en seis comunidades de la Costa de Oaxaca, y 162 productores maiceros en seis comunidades rurales de la Frailesca de Chiapas de Octubre a Diciembre de 2001.

El resto del estudio se divide en cinco secciones más: la segunda describe el método de análisis; la tercera ilustra someramente el área de estudio; la cuarta sección muestra la clasificación de los tipos de maíces así como los hallazgos de los materiales en las parcelas de los agricultores entrevistados en el trabajo de campo; la quinta muestra los resultados de la relación entre pobreza y consumo de maíz y en la sexta se realiza una discusión no concluyente del tema.

\section{2.- MÉTODO DE ANÁLISIS: LÍNEA MONETARIA DE POBREZA}

Una vez recabados los datos provenientes del trabajo de campo fue posible construir los perfiles de pobreza para cada comunidad, y a su vez para cada hogar, tomando como referencia el gasto per cápita mensual, para un mayor detalle sobre la decisión de considerar el gasto y no el ingreso ver Guevara et al. (2000).

La línea alimentaria de pobreza se estimó a partir de la canasta normativa alimentaria (CNA) diseñada por COPLAMAR en 1982. Para el propósito de este trabajo se decidió emplear la CNA de alimentos, que está integrada por 34 
productos alimenticios, que contienen 2,082 kilocalorías y 35.7 gramos de proteínas por adulto estimada por la Coordinación General del Plan Nacional para las Zonas Deprimidas y Grupos marginados (SEDESOL, 2002).

Para llegar a una estimación actualizada y completa de la línea monetaria de pobreza a partir de la CNA fue necesario realizar diversos ajustes, entre los que destaca actualizar el valor de la CNA para un hogar promedio en México (4.9 personas) el cual en enero de 1984 era de $\$ 121,403$ pesos corrientes. Si consideramos el incremento en el nivel general de precios desde ese año hasta noviembre de 2001, fecha en que se realizó la encuesta, se tendrá que multiplicar dicho valor por un factor de 161.052, ya que la inflación acumulada es de 16,005.19\%. Haciendo este ajuste, el valor anual de la CNA para noviembre de 2001 fue de $\$ 19,552$ nuevos pesos. Para obtener una cifra en términos mensuales y per cápita, se divide consecutivamente dicha cifra entre 12 (número de meses del año) y entre 4.9, que es el tamaño promedio de la familia. La cifra resultante es $\$ 351$ pesos, el método es tomado de Guevara et. al. 2000.

Para obtener los indicadores de pobreza en este estudio se eligió el indicador desarrollado por Foster, Greer y Thorbecke (1984), indicadores que se han consolidado como norma general en los estudios más recientes sobre pobreza. Dichos indicadores, que pertenecen a la familia de índices Pa, proporcionan varias ventajas porque a través de una sola cifra resumen y permiten identificar el nivel y la intensidad de la pobreza para todo un conjunto de población, y también para cada uno de los grupos que lo componen. Esto es posible porque dichos índices pueden desagregarse de forma aditiva, utilizando las proporciones poblacionales como ponderadores.

La derivación del indicador consiste en: sea z la variable que denota la línea alimentaria de pobreza, esto es, el ingreso mínimo requerido por una persona, dados sus patrones de preferencia en el consumo y de precios que paga para obtener una canasta de bienes (formada principalmente por alimentos) y así evitar grados de desnutrición (Gráfico 1).

La variable $G_{i}$ define la brecha de pobreza para la i-ésima persona de la población considerada como: 


$$
G_{i}=\operatorname{Max}\left[\left(z-y_{i}\right), 0\right]
$$

Donde $\mathrm{y}_{\mathrm{i}}$ presenta el ingreso per cápita de la i-ésima persona. Por tanto, la brecha tendrá valores positivos para toda persona por debajo de la línea de pobreza definida. A todas las demás personas se les asigna un valor de cero.

Para poder estimar el índice agregado de los índices Pa, se aplica el concepto de brecha individual a través de:

$$
P_{\alpha}(y ; z)=\frac{1}{n} \sum_{i=1}^{q}\left(\frac{G_{i}}{z}\right)^{\alpha}
$$

Donde q es el número de personas para las cuales $\mathrm{G}_{\mathrm{i}}>0$, es decir, para las personas que se encuentran por debajo de la línea de pobreza. El parámetro a representa la aversión social a la pobreza y n es el tamaño de la población. Cuando a es cero, $\mathrm{P}$ indica la proporción de la población que se encuentra por debajo de la línea de pobreza:

$$
P_{0}(y ; z)=\frac{1}{n} \sum_{i=1}^{q}\left(\frac{G_{i}}{z}\right)^{0}=\frac{q}{n}
$$

Sin embargo, aunque $\mathrm{P}_{\mathrm{o}}$ mide la extensión de la pobreza, no capta la severidad de la misma. No satisface el axioma de monotonicidad, que establece que "dadas otras cosas, una reducción en el gasto de los pobres debe incrementar la medida de pobreza". Cuando a $=1$, Pa satisface este axioma al convertirse en una medida sensitiva de la severidad de la pobreza. Cada una de las brechas de pobreza individuales se suma, encontrando así la brecha social relativa de pobreza:

$$
P_{1}(y ; z)=\frac{1}{n} \sum_{i=1}^{q}\left(\frac{G_{i}}{z}\right)^{1}
$$

$\mathrm{P}_{2}$, además de medir la severidad de la pobreza, permite considerar la desigualdad entre los pobres. Esto es posible porque este índice asigna una mayor ponderación a los más pobres entre los pobres. Además, $\mathrm{P}_{2}$ satisface el axioma 
de transferencia, que establece que "manteniendo todo lo demás constante, realizar una transferencia de una persona pobre a cualquier otra que es menos pobre, debe incrementar la medida de pobreza"1.

$$
P_{2}(y ; z)=\frac{1}{n} \sum_{i=1}^{q}\left(\frac{G_{i}}{z}\right)^{2}
$$

Una vez determinada la línea alimentaria de pobreza y haciendo explícitas las brechas relativas de la desigualdad entre los pobres, se modificaron los gastos de los hogares per cápita mensual por el factor de escala de equivalencia (Skoufias, 2002). El método de escala de equivalencias consiste en construir una escala de equivalencias que tome en consideración las distintas necesidades de los grupos por sexo y edad. El tamaño del hogar en unidades de adulto equivalente se construye usando distintas ponderaciones para los grupos de edad y sexo, de acuerdo con la definición proporcionada por el Instituto Nacional de Nutrición, 1987 (Skoufias, 2005). Específicamente, el tamaño de la familia por adulto equivalente (TFAE) se construye usando la siguiente fórmula:

TFAE $=(0.41)^{*}$ niños $0-4+(0.80)^{*}$ niños $5-10+(1.15)^{*}$ varones 11-14 + $(1.05)^{*}$ mujeres $11-14+(1.38)^{*}$ varones $15-19+(1.05)^{*}$ mujeres $15-19$ $+(1.26) *$ varones $20-34+(0.92) *$ mujeres $20-34+(1.15) *$ varones $35-54$ $+(0.85)^{*}$ mujeres $35-54+(1.03)^{*}$ varones $>=55+(0.78) *$ mujeres $>=55$.

\section{3.- ÁREA DE ESTUDIO: COSTA DE OAXACA Y FRAILESCA DE CHIAPAS}

Oaxaca y Chiapas son estados considerados entre las entidades federativas con mayores índices de pobreza y marginación, de acuerdo a los datos reportados por CONAPO-PROGRESA (2000) y las estimaciones de pobreza que

\footnotetext{
${ }^{1}$ Así como en todo índice de desigualdad subyacen juicios normativos (Cortés y Rubalcava, 1984; y García Rocha, 1986), P2 no queda exento de tal atributo, pues supone que, dada una cierta brecha social de pobreza, la igualdad entre los pobres es preferida. Es decir, la medida de pobreza se incrementa cuanto más marginadas se encuentren las personas en el extremo inferior de la distribución del ingreso.
} 
realizó el Comité Técnico para la Medición de la Pobreza (CTMP, 2002), así mismo, son de las entidades con mayor incidencia en desnutrición según la última encuesta nacional de nutrición (ENN, 1999) realizada por el Instituto Nacional de Nutrición (INN).

Seis comunidades están ubicadas en la región Costa de Oaxaca, y las restantes en la región Frailesca de Chiapas. Las 12 comunidades rurales fueron seleccionadas a partir de dos criterios básicos: por su nivel de marginación tomando como referencia el reporte de CONAPO-PROGRESA (2000), y la difusión de tecnología (transferencia de variedades mejoradas de maíz), la referencia fue el programa de Kilo por Kilo de la secretaría de agricultura <sagarpa.gob.mx $>$. Con esta base, se seleccionaron las comunidades, más detalles en Bellon et. al. (2003).

Las comunidades son: en la Costa de Oaxaca: San Pedro Jicayan, Santa María Magdalena Tiltepec, Santa María Cortijo, Santiago Jocotepec, Santos Reyes Nopala y San Pedro Mixtepec (Distrito-22); en la Frailesca de Chiapas: Dolores Jaltenango, Rizo de Oro, Roblada Grande, Querétaro, Primero de Mayo y Libertad Melchor Ocampo.

Los datos empleados en este análisis provienen de un estudio más amplio llevado a cabo en el Programa de Economía del Centro Internacional de Mejoramiento de Maíz y Trigo (CIMMYT), para mayor detalle ver Bellon et. al. (2003).

\section{4.- CLASIFICACIÓN DE LOS TIPOS DE MAÍZ}

El cuestionario aplicado a los hogares entrevistados integró una sección muy amplia sobre manejo de todos los tipos de maíz que el agricultor cultiva tanto en sus parcelas y el tras-patio, con ello, se obtuvo información de los diversos tipos de materiales que existen en cada hogar y en la comunidad. Los tipos de maíz se clasificaron en cuatro categorías, dependiendo del nombre que le otorga el agricultor, el número de años (o ciclos agrícolas) que el agricultor mantiene la misma semilla, y si la semilla fue comprada y salió originalmente de una bolsa (dado que la semilla nueva se vende en bolsa con tratamiento químico para ayudar a la germinación una vez que hace contacto con agua). 
Así entonces, los maíces se clasificaron en: (1) maíces criollos; (2) maíces acriollados [o generaciones avanzadas de maíces mejorados híbridos o de polinización abierta]; (3) variedades de polinización abierta (VPA); y (4) maíces híbridos. Además del cuestionario aplicado a los agricultores, en el trabajo de campo se realizaron dos grupos de enfoque y una colecta de maíz, con la finalidad de recolectar todos los tipos de maíces encontrados en cada comunidad.

La colecta de maíz ayudó a la identificación racial de los maíces, así como a la evaluación fenotípica y agronómica de cada maíz. Más detalles en Bellon et. al. (2003).

El número de maíces que cada agricultor maneja y conserva se muestran en el Cuadro 1, no es la finalidad de estudiar la adopción de tecnología o variedades mejoradas en este análisis, pero se puede apreciar que en la Frailesca de Chiapas, el 20\% de los hogares entrevistados usa maíces híbridos, y un 11\% de productores usa tanto maíces híbridos como criollos. No es materia de este análisis, pero el uso de más de un tipo de maíz responde a los atributos que cada material otorga a los agricultores, estos atributos pueden ser agronómicos, culinarios o de mercado, para abundar más ver Bellon et al. (2006). En el otro extremo, la mayoría de productores maiceros de Oaxaca conservan variedades criollas de maíz, cerca del 70\%, un porcentaje considerable, $18 \%$, recicla variedades modernas, o acriolladas, lo que probablemente muestra la dificultad por adquirir variedades modernas cada año o ciclo agrícola. O como lo demuestra Bellon et. al. (2006), las variedades mejoradas van adquiriendo atributos o rasgos - por consentimiento del agricultor- de características de las variedades criollas. Es decir, los agricultores mezclan atributos de cada tipo de maíz para reducir las diferencias entre tipos y obtener un maíz acorde a sus necesidades, proceso que toma muchos ciclos. Por último, el Cuadro 1 muestra que el uso de maíces híbridos en la Costa de Oaxaca es muy bajo, lo que puede sugerir que el acceso a la tecnología aún es muy distante, o que la tecnología no se ha hecho acorde a las condiciones ambientales (es decir, alta-pendiente, alta pedregocidad en las parcelas, alta-fragmentación de las parcelas, poca fertilidad en los suelos, entre otras características más) de la Costa Oaxaqueña. 


\section{5.- RESULTADOS}

De acuerdo con el Cuadro 2, existe mayor pobreza en la Costa de Oaxaca que en la Frailesca de Chipas, 67\% y 57\% respectivamente. En este mismo sentido, la severidad de la pobreza es mayor en la Costa que en la Frailesca con un $23 \%$ y un $18 \%$ para cada caso. Y como es de esperarse, la desigualdad se acentúa más en la Costa Oaxaqueña en un 10\%.

A pesar de que no es la finalidad en este estudio analizar la adopción de tecnología (considerado como adoptadores a los agricultores que usan algún material mejorado, sea maíz híbrido, de polinización abierta o acriollado), el Cuadro 2 también reporta que los agricultores que adoptan algún material mejorado son menos pobres que los que no adoptan, por ejemplo: los productores agrícolas en la Costa de Oaxaca que no adoptan maíz mejorado son más pobres que los que si lo hacen, $71 \%$ y $59 \%$ respectivamente. El mismo patrón se replica en la Frailesca de Chiapas.

Aún y el objetivo de este estudio no es hacer un análisis entre pobreza y grupos indígenas, el Cuadro 2, también aporta resultados para la Costa de Oaxaca sobre pobreza y pertenecer a un grupo indígena (Mixtecos mayoritariamente), así entonces, y de acuerdo a los resultados de las entrevistas y análisis respectivo: los grupos indígenas son más pobres en un $75 \%$ frente al grupo de mestizos con un 59\%, especial interés resalta la severidad y desigualdad para los grupos indígenas. Estos hallazgos son muy similares a la opinión de la gente expresada en los grupos de enfoque realizados en cada comunidad, donde hubo un consenso en el sentir de los participantes, donde el no hablar Español o sólo lengua indígena es una barrera sociocultural, que se traduce en pocas oportunidades de trabajo en el ámbito regional y nacional, de hecho hasta internacional (migración a los EE.UU.).

El Cuadro 3 reporta los resultados del gasto corriente que efectuaron los hogares en 2001 y su condición de pobreza. Para el caso de la Costa de Oaxaca, los hogares pobres destinan cerca del $40 \%$ de sus gastos totales en alimentos, y un poco más del $11 \%$ del gasto total corresponde a maíz en grano. Cantidad mucho mayor que sus vecinos en condición en ausencia de pobreza. Al respecto Morris (1998) comenta que entre mayor ingreso tenga un hogar, menor consumo y dependencia de maíz existe, es decir, después de cierto umbral de 
ingresos el hogar opta por consumir menos maíz y mayor carne y/o frutas y verduras. El Cuadro 4 muestra un patrón similar en la Frailesca, donde un poco más del $11 \%$ del gasto total que realizan los hogares pobres corresponde a maíz en grano.

Para ambas regiones, el gasto de aproximadamente $40 \%$ del total de erogaciones que realizan los hogares muestra, con claridad, que la mayoría realiza poca inversión en capital humano, financiero y gastos en salud, ocio y recreación, así como servicios básicos. Proporciones que muestran con claridad el bajo nivel de ingresos. Mucho se ha escrito sobre pobreza, formas y técnicas de medición, pero es suficiente y de sentido común analizar los rubros y proporciones de estos gasto de los hogares pobres del México rural, con una simple comparación, y debidas reglas de ajuste, es posible dilucidar la profundidad de pobreza en estas regiones.

\section{6.- DISCUSIÓN}

El análisis es somero y no concluyente, únicamente intenta demostrar la dependencia que los hogares pobres rurales guardan en el consumo de maíz en sus diferentes modalidades (tortillas elaboradas en casa con maíz de sus cosechas, tortillas elaboradas fuera del hogar [en tortillerías], harina de maíz comprada, y maíz en grano comprado, es decir, que no proviene de lo que cosecha el hogar). También, se muestra que los hogares no son autosuficientes en cuanto a maíz se refiere, pues la cosecha anual no alcanza para cubrir las necesidades del hogar. Así pues, en México existe un dilema entre producir maíz para la industria energética (elaboración de etanol) o producir maíz para alimentar a la población pobre del medio rural. También existe otro dilema, que es entre la conservación de biodiversidad de maíz y la adopción de variedades mejoradas de maíz, ya sean variedades de polinización abierta (VPA) o híbridos.

Es de suma importancia, en el ámbito federal, hacer política pública que mande señales de mercado afines para determinar el uso de la dotación nacional de tierras para cultivar maíz, pues producir maíz para energéticos que emplearían variedades de maíz amarillo, mayoritariamente para uso en la industria y se dejaría de producir maíz blanco para a alimentación humana. De 
acuerdo a las estadísticas oficiales, México es autosuficiente en la producción de maíz blanco, y deficitario en la producción de maíz amarillo, por tal motivo el gobierno se ha visto necesitado para ampliar los cupos de importación de maíz amarillo.

También es necesario realizar estudios más riguroso de los posibles escenarios de producir maíz para elaborar etanol, así como la factibilidad y viabilidad técnica en un país, como México, donde el cereal es el sustento de la alimentación de millones de habitantes. Así pues, el propósito de este análisis es abrir más preguntas de investigación, que motiven futuras investigaciones.

\section{BIBLIOGRAFÍA}

Aquino, P. (1998). Mexico. Maize seed industries in developing countries. M. L. Morris. Colorado, Rienner \& CIMMYT: 401.

Bellon, M. R. and S. B. Brush (1994). "Keepers of Maize in Chiapas, Mexico." Economic Botany 48(2): 196-209.

Bellon, M. R., Michelle Adato, Javier Becerril y Dubravka Mindek (2006). "Poor Farmers' Perceived Benefits from Different Types of Maize Germplasm: The Case of Creolization in Lowland Tropical Mexico." World Development 34(1): 113-129.

Bellon, M. R., Michelle Adato, Javier Becerril y D. Mindek (2003). The impact of improved maize germplasm on poverty alleviation: the case of Tuxpeño-derived material in Mexico. Washington, D. C., FCND Discussion Paper 162, CIMMYT \& IFPRI: 46.

Brush, S. B. (1986). "Genetic diversity and conservation in traditional farming systems." Journal of Ethnobiology 6(1): 151-167.

CONAPO-PROGRESA (2000). Indices de marginación 1995. Mexico, D. F., Consejo Nacional de Población y Programa de Educación, Alimentación y Salud: 160. 
CTMP (2002). (Comité Técnico para la Medición de la Pobreza CTMP) Medición de la pobreza: variantes metodológicas y estimación preliminar. Mexico D.F, Secretaría de Desarrollo Social.

Dyer, G. y A. Yunez-Naude (2003). NAFTA and conservation of maize diversity in Mexico. Mexico City, Commission for Environmental Cooperation: 29.

Foster, J., J. Geer y Erik Thorbecke (1984). "A class of decomposable poverty measures." Econometrica 52(3): 761-765.

Guevara, A., Carlos Muñoz-Piña, Gabriela Estrada-Díaz y N. Acosta-Romero (2000). Manual para la evaluación de impactos sobre el abatimiento de la pobreza a partir de la inversión en proyectos ambientales en pequeñas poblaciones rurales. Mexico D. F., Universidad Iberoamericana Serie de Trabajo 500-03.

Morris, M. L. (1998). 2 Overview of the world maize economy. Maize seed industries in developing countries. M. L. Morris. Colorado, Rienner \& CIMMYT: 401.

Morris, M. L. (1998a). Maize seed industries in developing countries. Colorado, Rienner \& CIMMYT.

Morris, M. L. y M. A. López-Pereira (1999). Impacts of maize breeding research in Latin America, 1966-1997. México D. F., CIMMYT: 45.

Perales, H., S. B. Brush y C. O. Qualset (2003a). "Landraces of maize in central Mexico: an altitudinal transect." Economic Botany 57(1): 7-20.

Perales, H., S. B. Brush y C. O. Qualset (2003b). "Dynamic management of maize landraces in central Mexico." Economic Botany 57(1): 21-34.

SEP (2002). El Maíz: fundamento de la cultura mexicana (exposición). México, Secretaria de Educación Pública (SEP), Museo Nacional de Culturas Populares. García \& Valdés Editores S. A. 
SIAP (2007). Situación actual y perspectivas del maíz en México 1996-2012. Mexico D.F., Servicio de Información Agroalimentaria y Pesquera (SIAP) SAGARPA: 208.

Skoufias, E. (2005). PROGRESA and its impacts on the welfare of rural households in Mexico. Washington, International Food Policy Research Institute: 84 .

Yunez-Naude, A. (2003). "The dismantling of CONASUPO, a Mexican state trader in Agriculture." The World Economy 26(1): 97-122. 


\section{ANEXOS}

Gráfica 1

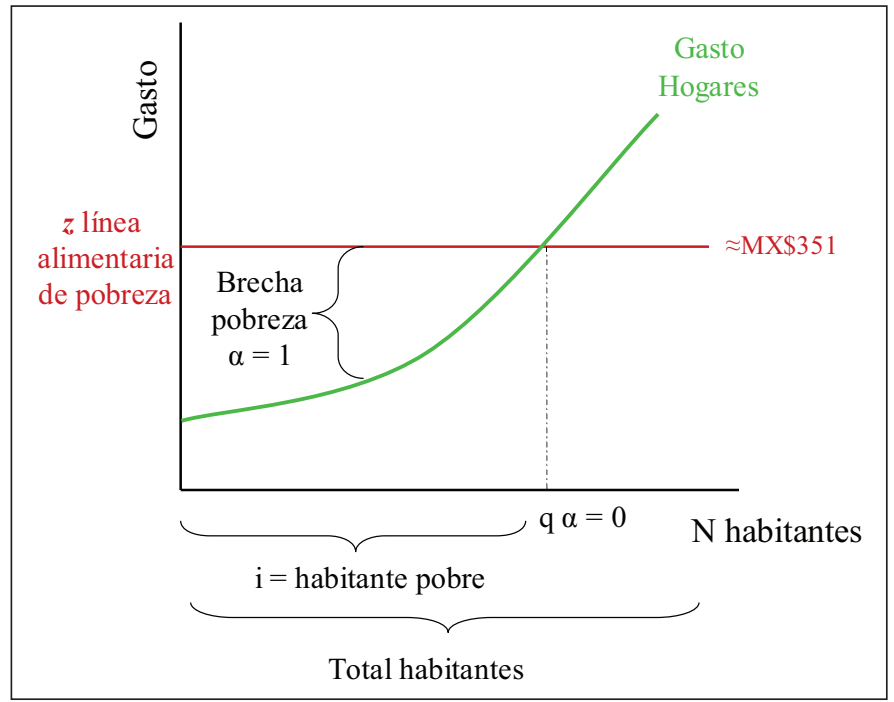

Fuente: Guevara et. al 2000.

Cuadro 1

Maíces en manos de los agricultores

\begin{tabular}{|l|c|c|c|c|}
\hline \multirow{2}{*}{ Todas las combinaciones de Maíz } & \multicolumn{4}{|c|}{ Frecuencia (a) } \\
\cline { 2 - 5 } & Chiapas & $\%$ & Oaxaca & $\%$ \\
\hline Híbrido & 33.0 & 20.4 & 4.0 & 2.5 \\
\hline Híbrido \& Criollo & 19.0 & 11.7 & 7.0 & 4.3 \\
\hline Híbrido \& Acriollado & 10.0 & 6.2 & - & - \\
\hline Híbrido, Acriollado \& Criollo & 3.0 & 1.9 & - & - \\
\hline Híbrido \& VPA & 8.0 & 4.9 & - & - \\
\hline Híbrido, VPA \& Criollo & 3.0 & 1.9 & - & - \\
\hline Híbrido, VPA \& Acriollado & 1.0 & 0.6 & - & - \\
\hline Todos los tipos & - & - & - & - \\
\hline Criollo & 32.0 & 19.8 & 111.0 & 68.1 \\
\hline Acriollado & 22.0 & 13.6 & 30.0 & 18.4 \\
\hline Acriollado \& Criollo & 2.0 & 1.2 & 9.0 & 5.5 \\
\hline VPA* & 23.0 & 14.2 & 2.0 & 1.2 \\
\hline VPA \& Criollo & 3.0 & 1.9 & - & - \\
\hline VPA \& Acriollado & 3.0 & 1.9 & - & - \\
\hline VPA, Acriollado \& Criollo & - & - & - & - \\
\hline (a) Número de Hogares & $\mathrm{N}=162$ & 100 & $\mathrm{~N}=163$ & 100 \\
\hline * Variedad de Polinizaćn Abierta & & & & \\
\hline
\end{tabular}

* Variedad de Polinización Abierta 
Cuadro 2

Indicadores de pobreza: Foster, Greer and Thorbecke (1984)

\begin{tabular}{|c|c|c|c|c|}
\hline & \multicolumn{2}{|c|}{ Oaxaca todos Hh's } & \multicolumn{2}{|c|}{ Chiapas Todos Hh's } \\
\hline Proporción Pobreza $\alpha=0$ & \multicolumn{2}{|c|}{0.672} & \multicolumn{2}{|c|}{0.566} \\
\hline Severidad Pobreza $\alpha=1$ & \multicolumn{2}{|c|}{0.230} & \multicolumn{2}{|c|}{0.180} \\
\hline Desigualdad Pobres $\alpha=2$ & \multicolumn{2}{|c|}{0.109} & \multicolumn{2}{|c|}{0.080} \\
\hline & Adopción & No-Adopción & Adopción & No-Adopción \\
\hline Proporción Pobreza $\alpha=0$ & 0.595 & 0.708 & 0.543 & 0.648 \\
\hline Severidad Pobreza $\alpha=1$ & 0.229 & 0.231 & 0.168 & 0.219 \\
\hline \multirow[t]{2}{*}{ Desigualdad Pobres $\alpha=2$} & 0.111 & 0.108 & 0.075 & 0.100 \\
\hline & No-indígena & Indígena & & \\
\hline Proporción Pobreza & 0.589 & 0.750 & & \\
\hline Severidad Pobreza & 0.195 & 0.263 & & \\
\hline Desigualdad Pobres & 0.088 & 0.128 & & \\
\hline
\end{tabular}

Fuente: información trabajo de campo 2001.

Cuadro 3

Oaxaca Gasto Hogares 163 Hh's

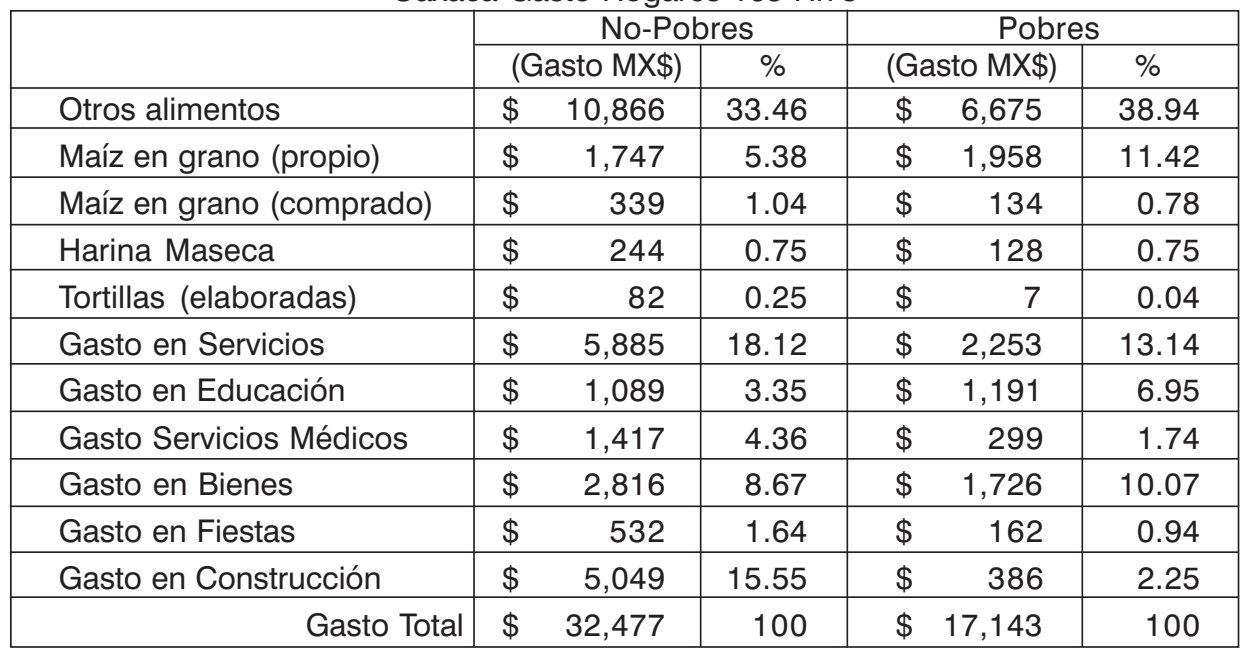

$M X \$ 2001$, US\$1 $=M X \$ 9.32$ 
Cuadro 4

Chiapas Gasto Hogares 162 Hh's

\begin{tabular}{|c|c|c|c|c|c|c|}
\hline & \multicolumn{3}{|c|}{ No Pobres } & \multicolumn{3}{|c|}{ Pobres } \\
\hline & \multicolumn{2}{|c|}{ (Gasto MX\$) } & \multirow{2}{*}{$\begin{array}{c}\% \\
40.91\end{array}$} & \multicolumn{2}{|c|}{ (Gasto MX\$) } & \multirow{2}{*}{$\begin{array}{c}\% \\
41.53\end{array}$} \\
\hline Otros alimentos & $\$$ & 11,887 & & $\$$ & 7,745 & \\
\hline Maíz en grano (propio) & $\$$ & 1,438 & 4.95 & $\$$ & 2,227 & 11.94 \\
\hline Maíz en grano (comprado) & $\$$ & 58 & 0.20 & $\$$ & 2 & 0.01 \\
\hline Harina Maseca & $\$$ & 243 & 0.84 & $\$$ & 51 & 0.27 \\
\hline Tortillas (elaboradas) & $\$$ & 886 & 3.05 & $\$$ & 93 & 0.50 \\
\hline Gasto en Servicios & $\$$ & 4,519 & 15.55 & $\$$ & 2,190 & 11.74 \\
\hline Gasto en Educación & $\$$ & 916 & 3.15 & $\$$ & 821 & 4.40 \\
\hline Gasto Servicios Médicos & $\$$ & 1,697 & 5.84 & $\$$ & 168 & 0.90 \\
\hline Gasto en Bienes & $\$$ & 3,464 & 11.92 & $\$$ & 2,884 & 15.46 \\
\hline Gasto en Fiestas & $\$$ & 434 & 1.49 & $\$$ & 58 & 0.31 \\
\hline Gasto en Construcción & $\$$ & 894 & 3.08 & $\$$ & 40 & 0.21 \\
\hline Gasto Total & $\$$ & 29,059 & 100 & $\$$ & 18,650 & 100 \\
\hline
\end{tabular}

$M X \$ 2001$, US\$1 = MX\$9.32 